\title{
High count rate pulse shape discrimination algorithms for neutron scattering facilities
}

\author{
S. Richards, G. J. Sykora, M. P. Taggart
}

\section{Published version information}

Citation: S Richards, G Sykora and M Taggart. 'High count rate pulse shape discrimination algorithms for neutron scattering facilities.' Nucl Instrum Meth A, vol. 989 (2021): 164946.

DOI: $\underline{\text { 10.1016/i.nima.2020.164946 }}$

(C)2020. This manuscript version is made available under the CC-BY-NC-ND 4.0 Licence.

This version is made available in accordance with publisher policies. Please cite only the published version using the reference above. This is the citation assigned by the publisher at the time of issuing the AAM. Please check the publisher's website for any updates. 


\title{
High Count Rate Pulse Shape Discrimination Algorithms for Neutron Scattering Facilities
}

\author{
S. Richards ${ }^{1}$, G. J. Sykora ${ }^{2}$, M. P. Taggart ${ }^{3}$ \\ [1] Detector Development Group - Science and Technology Facilities Council, Rutherford Appleton Laboratory, Harwell Science and \\ Innovation Campus, Didcot, Oxfordshire, OX11 0QX. These authors contributed equally to this work. [2] ISIS Detector Group - Science and \\ Technology Facilities Council, Rutherford Appleton Laboratory, Harwell Science and Innovation Campus, Didcot, Oxfordshire, OX11 0QX. \\ [3] Department of Physics, University of Surrey, Guildford, Surrey, GU2 7XH
}

\begin{abstract}
The performance of EJ-270, a ${ }^{6} \mathrm{Li}$ loaded pulse shape discriminating plastic scintillator was tested for use in applications with high thermal and epithermal neutron fluxes such as neutron scattering facilities. The short decay time of EJ-270 make it of interest for high count rate applications. To realize this, 4 PSD algorithms were tested. The algorithms were selected based on the potential for high rate applications and simplicity. These algorithms were the charge integration method with and without the addition of a digital low pass filter, a measurement of the time to $10 \%$ of peak amplitude and a method we call the "tail sum" which utilizes a digital low pass filter and sums a small number of samples in the tail of each pulse. These algorithms were benchmarked using the figure of merit, the $\gamma$-sensitivity and potential rate capability. The charge integration method gave the highest figure of merit of 1.37 using a long window of $272.5 \mathrm{~ns}$ but had a $\gamma$-sensitivity of $2 \times 10^{-6}$ which was poorer than the tail sum algorithm. The tail sum algorithm was able to achieve a figure of merit of 1.36 with a window of $250 \mathrm{~ns}$ and a $\mathrm{y}$-sensitivity on the order of $10^{-7}$. Reducing the integration windows to match the fastest algorithm of time to $10 \%$ resulted in the tail sum outperforming the other algorithms with a figure of merit of 1.26 and a $Y$-sensitivity of $6 \times 10^{-7}$. The short charge integration method and tail-sum were compared on the EMMA beamline at the ISIS pulsed neutron and muon source. The tails sum demonstrated better separation between the $\gamma$-rays and thermal neutron at an incident peak neutron rate of $9.6 \times 10^{5}$ neutrons per second.
\end{abstract}

Keywords: EJ-270, Scintillator, Neutron Scattering, Thermal Neutron Detection, Pulse Shape Discrimination.

\section{Introduction}

Spallation neutron sources are an important tool for material science. The use of thermal and epithermal neutrons from these sources enable techniques such as powder and single crystal diffraction, neutron reflectometry, small angle scattering and imaging. Next generation sources such as the European Spallation Source and upgrades to current facilities such as J-Parc, the Spallation Neutron Source, and the ISIS pulsed neutron and muon source will generate high neutron fluxes which pose challenges to current detector technologies [1]. The scintillation detectors ISIS currently utilises are cerium doped ${ }^{6} \mathrm{Li}$-glass and $\mathrm{ZnS}: \mathrm{Ag} /{ }^{6} \mathrm{LiF}$ scintillators. However, the glass suffers from high $\mathrm{Y}$-ray sensitivity and ZnS:Ag has a long afterglow which limits its applications at high rate [2]. The recently developed ${ }^{6} \mathrm{Li}$-loaded plastic scintillator, EJ-270, is of interest owing to the fast decay, the inherent low $\gamma$-sensitivity, and pulse shape discrimination (PSD) properties. 
The ${ }^{6}$ Li containing EJ-270 plastic scintillator, developed by Eljen Technologies and Lawrence Livermore National Laboratories, exhibit pulse shape discrimination of $\gamma$-ray and charged particle events [3, 4]. Typical of polyvinyl toluene (PVT) based scintillators, EJ-270 is low cost, available in large volumes and, despite fogging in certain environments, maintains good environmental stability [5]. These attributes make EJ-270 an attractive alternative to other fast PSD scintillators such as CLYC $[6,7]$ particularly in applications where good energy resolution is not a requirement.

Pulse shape discriminating scintillators are popular for applications in homeland security where special nuclear materials such as ${ }^{235} \mathrm{U},{ }^{233} \mathrm{U}$ and ${ }^{239} \mathrm{Pu}$ emit neutrons during spontaneous fission events. Detectors capable of discriminating neutrons from $y$-ray events allow for the neutrons emitted from these materials to be detected with high confidence. The research effort in this field has focussed on utilising PSD for detecting fast neutrons $\left(E_{k}>1 M e V\right)$ in plastic scintillators $[8,9,10$, 11]. The key differences between the homeland security applications and neutron scattering are the energy and flux of the incident radiation. For neutron scattering only epithermal, thermal and cold neutrons are of interest. Sensitivity to these neutron energies is provided through the addition of ${ }^{6} \mathrm{Li}$ to the plastic base polymer. The neutron flux is significantly higher at a spallation neutron source than would be expected from an illicit sample of special nuclear materials leading to pulse pile up. Consequently, the fast decay time of EJ-270 makes it attractive for high rate applications such as single point detectors for reflectometry, small angle neutron scattering, or beamline diagnostics. A short decay PSD scintillator such as EJ-270 would be needed for these applications along with the ability to perform pulse shape discrimination at high rates in real time.

A popular PSD algorithm is the charge comparison algorithm (discussed in more detail in section 3 ) [12]. This algorithm integrates the pulse from the scintillator using short and long time windows, the ratio between the long and short window gives a PSD parameter (Eq. 3) which can be used to discriminate neutron events from $\gamma$-ray events $[3,4]$. For EJ-270 coupled to a photomultiplier tube (PMT), the long window time reported in the literature is $500 \mathrm{~ns}$ [4]. The decay of EJ-270 is however much faster. The time to $10 \%$ from peak amplitude is $\sim 50-70$ ns depending on the exciting particle type $[3,4]$. Therefore, an alternative to the charge integration algorithm utilizing the fast decay time could allow for higher count rates. While there are PSD algorithms found in the literature that can operate on very short sample lengths, such as the simplified digital charge collection algorithm, they usually rely on functions that are resource intensive for an FPGA [13]. The two high rate PSD algorithms presented in this work are "time to 10\%" - a measurement of the delay between the peak amplitude of the pulse and its decay to $10 \%$ of that value, and "tail sum" - an integral across a small number of samples in the tail of the pulse.

Additional challenges to neutron scattering applications are that detectors are typically multichannel, large area and the physical constraints of the experimental setup typically results in long cables between detector elements. To achieve this, an algorithm capable of real time discrimination at high rates would be needed. An algorithm that is simple to implement on a FPGA would not only allow for minimal processing time in the FPGA but would also allow for more channels per FPGA, reducing the cost of large multi-channel systems or the use of more affordable FPGAs for smaller single channel systems.

\section{Materials and Method}


Two sets of experiments were performed to characterize EJ-270 and the PSD algorithms. The first set was algorithm development and testing using sealed sources and the second was count rate tests at the EMMA beamline at the ISIS Pulsed Neutron and Muon source [14].

\subsection{Detector configuration}

The detector consisted of a $48 \mathrm{~mm}$ diameter $x 15 \mathrm{~mm}$ thick cylinder of EJ-270 (shown in Fig. 1a) coupled to a Hamamatsu R6231-100 PMT using Dow Corning Vacuum grease. The scintillator was wrapped in PTFE tape to enhance light collection. The coupled EJ270/PMT system was encased in a light-tight aluminum enclosure. A negatively biased E1198-26 base at -1000 V was used to avoid signal reduction effects due to charge build up on the decoupling capacitor of positively biased bases such as those typically used with PSD plastic scintillators. A $6 \mathrm{~m}$ long coaxial cable was used from the PMT to the digitizer. Testing on the EMMA beamline (described below) required a $30 \mathrm{~m}$ long cable due to space restrictions on the beamline. Signals from the PMT were digitized using an Acqiris 400 MSps 12-bit digitizer with trace lengths of $500 \mathrm{~ns}$. Traces were stored for off-line processing using Python.

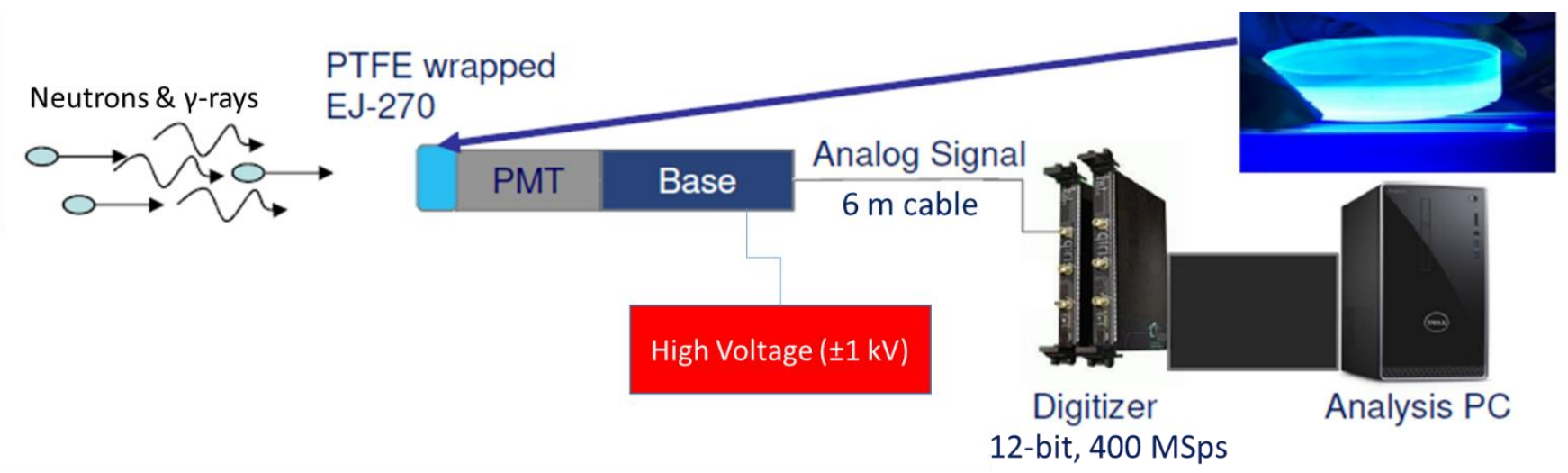

Figure 1. The schematic of the detector configuration.

\subsection{Irradiation conditions}

Pulse shape discrimination algorithms were tested using measurements from a water moderated ${ }^{241} \mathrm{Am}-\mathrm{Be}$ source ( $370 \mathrm{GBq}$ activity) shielded with lead. The detector was placed $50 \mathrm{~cm}$ from the source providing a uniform neutron flux of approximately 100 neutrons per second per $\mathrm{cm}^{2}$. Gamma sensitivity was assessed by placing the detector $30 \mathrm{~cm}$ from a $7.4 \mathrm{MBq}{ }^{60} \mathrm{Co}$ source.

The performance of EJ-270 as a function of neutron flux was tested on the EMMA beamline at the ISIS Pulsed Neutron and Muon source. EMMA has a direct line of site to the room temperature water moderator on ISIS. The instrument flight path is $15.6 \mathrm{~m}$ long. Neutrons are in a range from approximately $500 \mathrm{meV}(0.4 \AA)$ to $3.25 \mathrm{meV}(5 \AA)$ with a peak energy of $80 \mathrm{meV}$ ( $1 \AA$ in wavelength). Higher energy neutrons are chopped out and further discriminated by time of flight. All measurements presented here were time gated to measure a neutron energy range of $75 \mathrm{meV}-90$ $\mathrm{meV}$. The neutron flux on EMMA at $80 \mathrm{meV}$ is $1 \times 10^{6}$ neutrons per second per $\mathrm{cm}^{2}$. The overall incident flux on the detector was controlled by a set of cadmium slits upstream of the detector. 


$$
P S D_{T S}=\frac{1}{V_{p}} \sum_{S t=T_{D 1}}^{T_{D 1+i}} V_{S t}
$$
also represented in figure 2 . All three methods are compared here. different percentage could be used with other scintillators. sum with pulse height to be accounted for (Eq. 4).

Two alternative PSD methods investigated here are a time to $10 \%$ of the pulse height and integrating charge in only a short window in the tail of the signal (referred to as the tail sum). Both methods are

The time to $10 \%$ algorithm is conceptually the simplest of the three tested algorithms. A neutron event has a longer decay and therefore discrimination can be identified by its longer time to $10 \%$. This specific percentage was selected based on study of the pulse shapes and basic optimisation, a

The tail sum algorithm consists of summing the amplitude, $V_{s t}$, from the digitizer over a set number of samples, $i$, (analogous to window length) starting a time, $t_{D 1}$, after the peak. Then this sum is normalized to the peak amplitude of the pulse (explained below), $V_{p}$, allowing for the increase in tail 


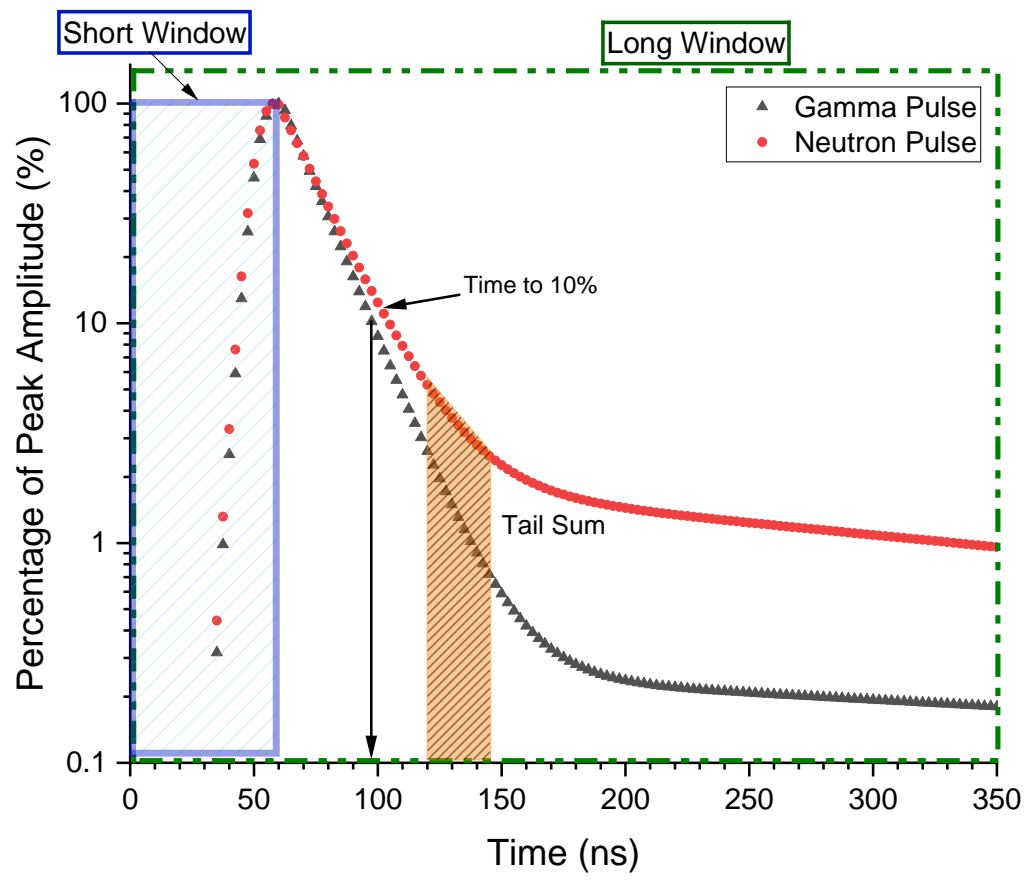

141

142

Figure 2. The schematic of typical pulses from a pulse shape discriminating scintillator showing how $\psi$-rays and neutrons differ and showing the three PSD algorithms. The window position are representative and do not show the exact window length and position of each algorithm.

The digitized pulses were processed using Python. For the sealed source data each trace contained only one pulse allowing the numpy max function to accurately report the peak amplitude [15]. The traces from the EMMA instrument contained multiple pulses and therefore the peak finding function in the peakutils library was used. The pulse shape discrimination was performed using the time to $10 \%$ algorithm, the tail sum algorithm and the charge integration algorithm with and without a digital low pass filter. The filter from the scipy library was a first order digital low pass Bessel filter with a $10 \mathrm{MHz}$ cut off frequency, it was used with the tail sum and time to $10 \%$ algorithms [16]. The charge integration algorithm was tested with and without the low pass Bessel filter. No other processing such as baseline correction was applied to the data. While a DC offset was present no shift in the offset was observed. As we were focused on the thermal neutron peak the lack of offset removal did not influence the charge integration algorithm results. A basic energy calibration was performed based on the Compton edges from the 1.17 and $1.33 \mathrm{MeV}{ }^{60} \mathrm{Co}$ gamma rays and the 480 keV gamma ray emitted by the ${ }^{10} \mathrm{~B}$ neutron capture reaction.

The most common method for quantifying the performance of a PSD based detector is the Figure of Merit (FoM) [17]. The FoM (Eq. 5) is determined for a given energy range by plotting the distribution of the pulse shape parameter and calculating the separation of the peaks and their full widths at half maximum.

$$
F O M=\frac{\text { Centroid }_{n}-\text { Centroid }_{\gamma}}{F W H M_{n}+F W H M_{\gamma}}
$$

Typically the FoM will be calculated from data acquired on a sealed source such as an AmericiumBeryllium or Californium sources, both of which can be moderated to produce thermal neutrons. While this method of quantification works well for homeland security, it has limitations for neutron 
scattering instrumentation. The $\gamma$-ray background at a neutron scattering experiment changes with the experimental setup. Different samples produce different backgrounds, the use of boron, cadmium or gadolinium based shielding and beam jaws also change the background, as such the $\gamma$ ray sensitivity is a preferred measure of discrimination.

The $\gamma$-ray sensitivity measurement is performed using a prolonged measurement on a pure $\gamma$-ray source such as ${ }^{60} \mathrm{Co}$. After background subtraction, the ratio between the number of incident $\gamma$-rays and the number of events that the system identifies as neutrons gives the $\psi$-ray sensitivity $[18,19]$. This is effectively a false positive rate.

An optimization script iterated the start and end points of the integration windows for both forms of the charge integration and the single window for the tail sum. The script measured the gamma sensitivity and the figure of merit for the energy range corresponding to \pm 2 standard deviations $(\sigma)$ from the centroid of the neutron capture peak for each possible window combination. The optimization of time to $10 \%$ was basic and not as exhaustive as the other optimizations. The script measured the figure of merit and gamma sensitivity using the same methodology as the other algorithms but the tested values ranged from time to $15 \%$ to time to $5 \%$ in steps of $1 \%$.

The optimization for count rate limited the maximum value of the window to match the duration of the time to $10 \%$ algorithm.

The performance of the algorithms were evaluated by comparing the FoM, the sensitivity to ${ }^{60} \mathrm{Co} \mathrm{Y}$ ray in the region corresponding to the ${ }^{6} \mathrm{Li}$ neutron capture peak, and the duration of the measurement to achieve discrimination.

\section{Results and Discussion}

\subsection{Optimizing processing parameters - Sealed Source Results}

2D histograms of gamma equivalent energy vs. PSD parameter for each tested algorithm from data taken on the moderated ${ }^{241} \mathrm{Am}$-Be source are shown in figure 3 . The figures show the characteristic thermal neutron capture peak at $\sim 300-400$ keVee which overlays the continuum of fast neutrons, at a lower PSD value, the continuum from $\psi$-rays is observed. All 4 algorithms show some separation between the $\gamma$-rays and the thermal neutron peak. This separation is better demonstrated in figure 4, which shows the distribution of the PSD parameter for each tested algorithm, at the energy range corresponding to thermal neutron events. The figure of merit is calculated using equation 5 after fitting each distribution using a double Gaussian function. For both figures the sub-figures labelled " $\mathrm{A}$ " are the standard charge integration method, " $\mathrm{B}$ " corresponds the charge integration algorithm but after applying a digital low pass filter to the traces, " $C$ " shows the tail sum results and $D$ is the time to $10 \%$. 

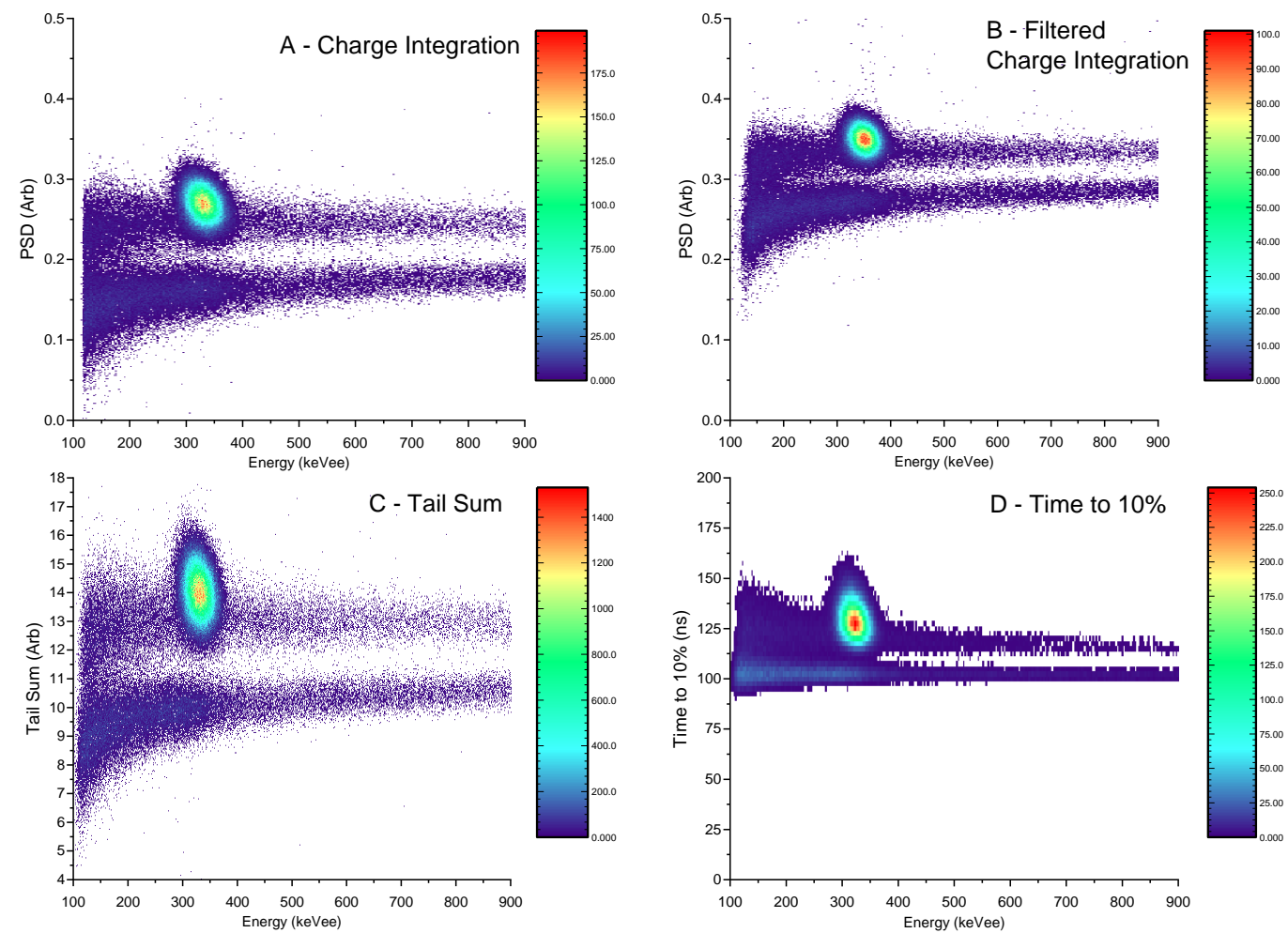

Figure 3. 2D PSD histograms for A: charge integration, B: Filtered Charge integration, C: Tail sum, D:

Time to $10 \%$.
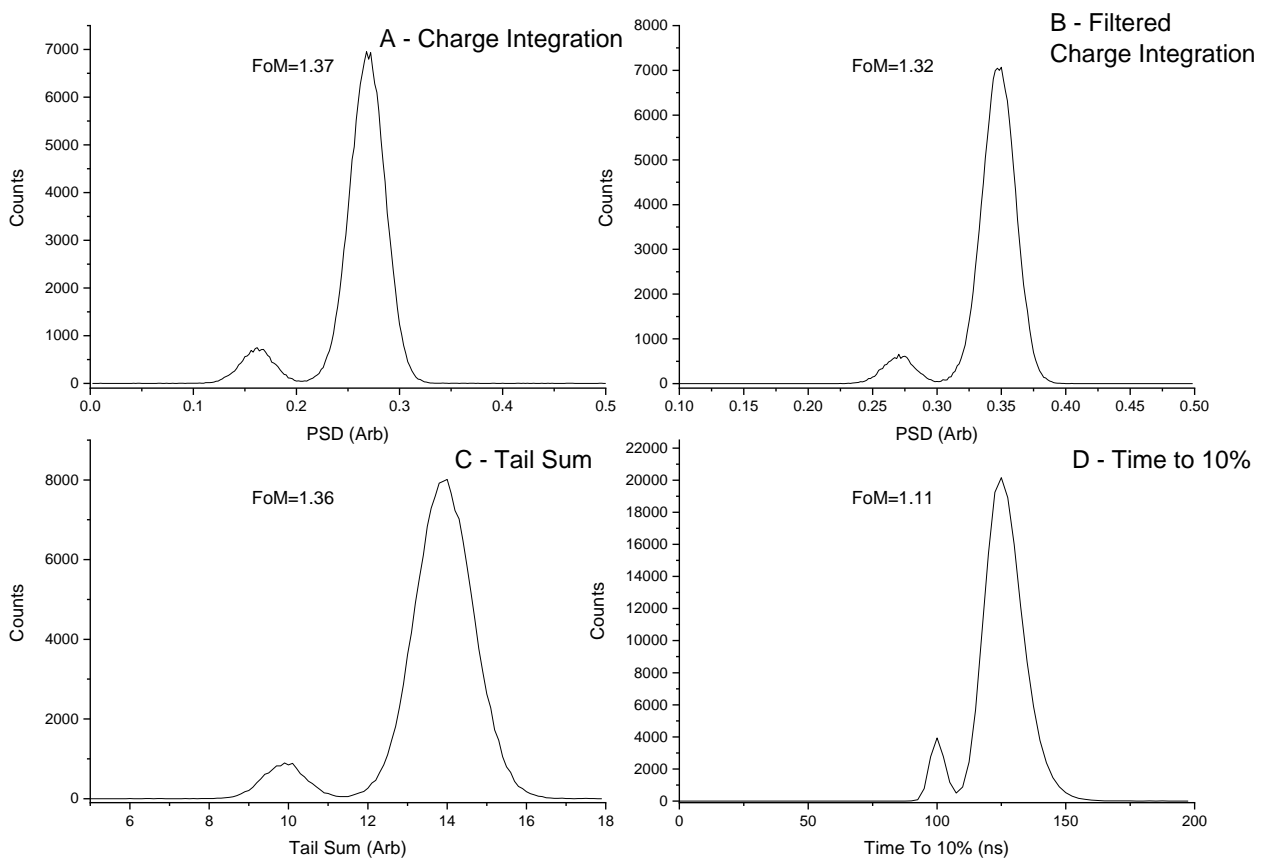

Figure 4. Histograms of counts as a function of PSD parameter for an energy range corresponding to the thermal neutron peak ( $270 \mathrm{keVee}$ to $350 \mathrm{keVee}$ ) and their associated figure of merit for- $\mathrm{A}$ : charge integration, B: Filtered Charge integration, C: Tail sum and D: Time to $10 \%$. 
While fast neutrons are not the focus of this study, figures of merit for the best performing settings of each different algorithms were plotted for 4 energy ranges as shown in figure 5 . The Tail Sum algorithm achieved the highest figure of merit. Figure of merit is seen to increase in general as the energy increases for all PSD algorithms. Higher energy recoil protons and gammas result in overall brighter events and better photon statistics accounting for improved figure of merit at higher energies. A few exceptions occur around the 650-700 keVee range where the high rate algorithms reduce slightly before increasing again. The exact cause of the reduced figure of merit is not yet known, however the effect is minor and should not drastically affect overall performance.

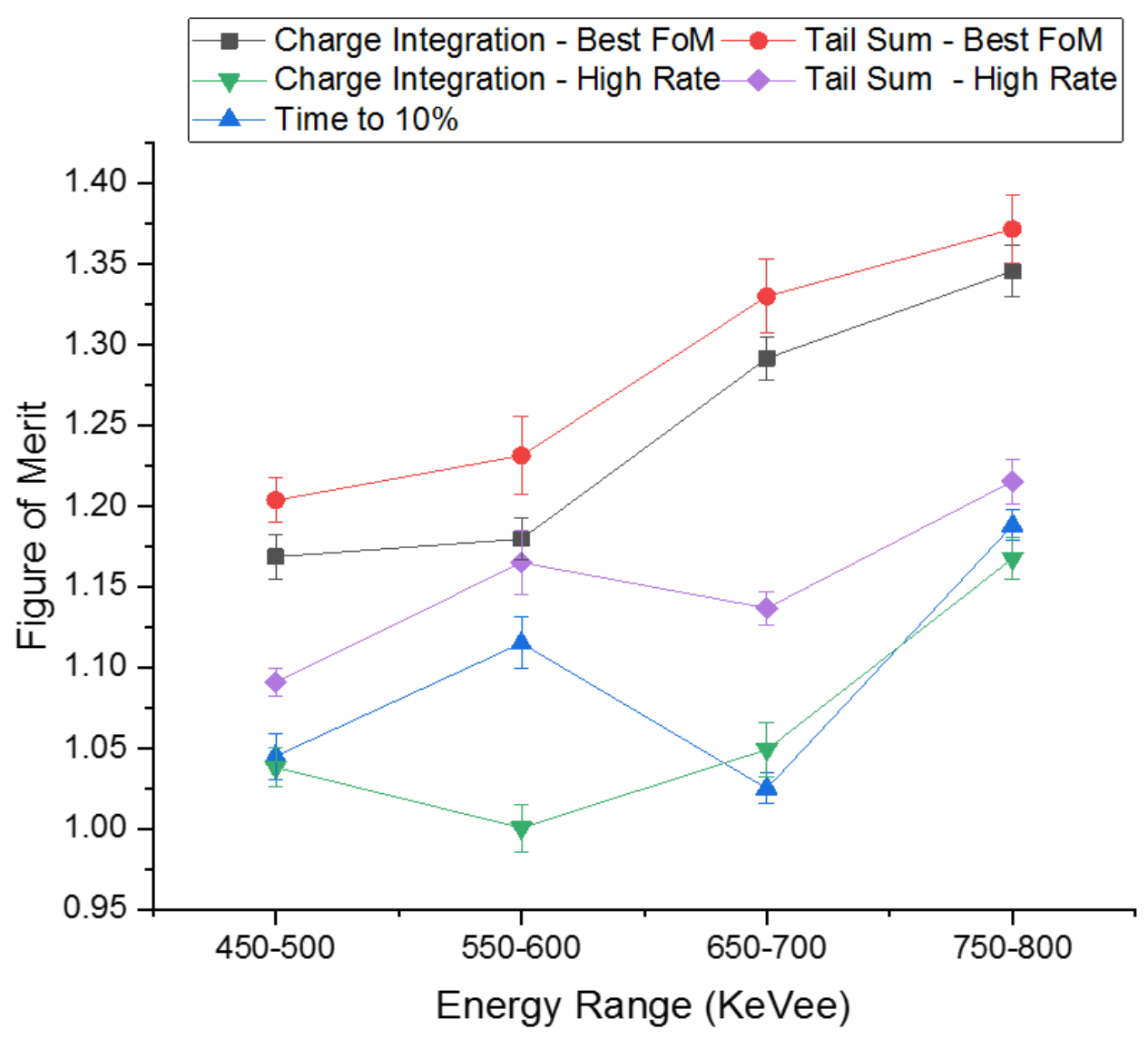

Figure 5. The figure of merit as a function of electron equivalent energy for fast neutrons present in the ${ }^{241} \mathrm{Am}$-Be measurements for each tested algorithm.

The $\gamma$-sensitivity of the EJ-270 was measured using a moderated ${ }^{241} \mathrm{Am}-\mathrm{Be}$ source and a ${ }^{60} \mathrm{Co}$ source. The location of the neutron capture peak in terms of PSD and pulse height was determined using the moderated ${ }^{241} \mathrm{Am}-\mathrm{Be}$ source. $\mathrm{A} \pm 2 \sigma$ boundary around the centroid of the neutron peak's pulse height was used and $a+2 \sigma$ and $-1.6 \sigma$ boundary around the centroid of the neutron peak's PSD value. These boundaries were then applied to ${ }^{60} \mathrm{Co}$ results along with a background subtraction, resulting in the $\gamma$ sensitivity factor (Figure 6). 
A)

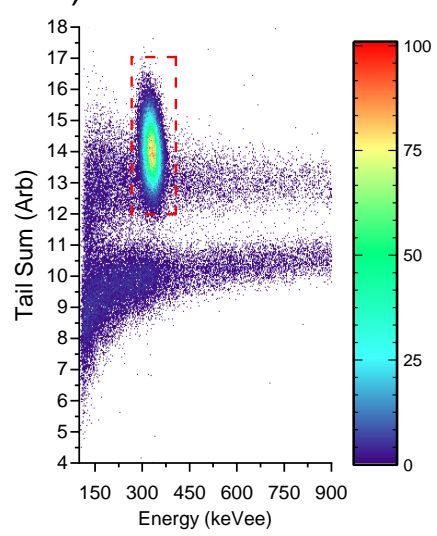

B)

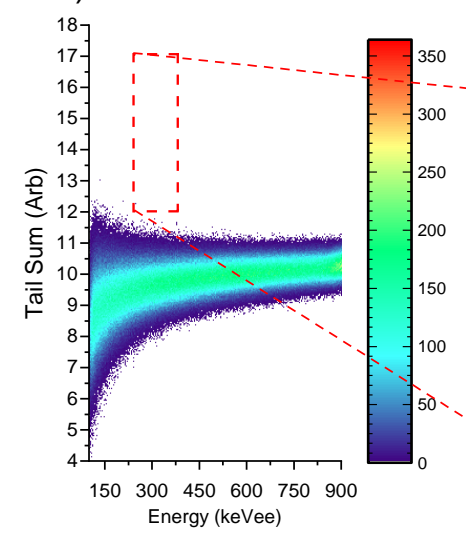

C)

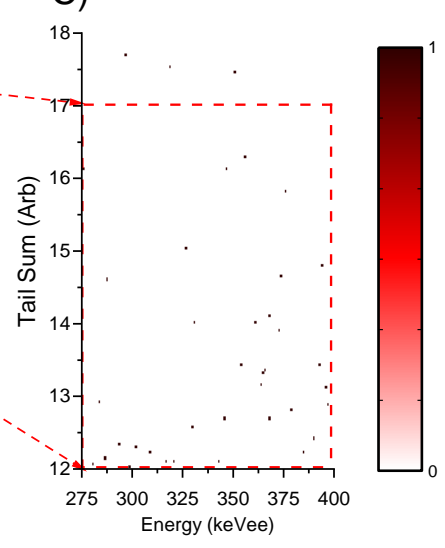

Figure 6. 2D histograms showing PSD from A) a moderated ${ }^{241} \mathrm{Am}-\mathrm{Be}$ source $\mathrm{B}$ ) plot from a ${ }^{60} \mathrm{Co}$ source, and C) a zoom in of the region where thermal neutrons would appear. The features in A) are thermal neutrons (highlighted by the box) and two horizontal bands; the lower band are $\mathrm{Y}$-ray events as they have faster decays and the upper band are fast neutron events.

The differences in the separation of the ${ }^{6} \mathrm{Li}$ capture peak and the $\mathrm{\gamma}$-ray background are evident in all four plots. The addition of the low pass filter to the charge integration method resulted in a factor of 2.5 worse gamma sensitivity and reduction of $4 \%$ in figure of merit. The charge integration method resulted in the best figure of merit of 1.37 but also a 22.5 ns longer measurement duration. Despite the marginally lower figure of merit than the charge integration method, the tail sum method resulted in the best $\nu$-sensitivity of approximately $10^{-7}$ counts per gamma. The time to $10 \%$ method resulted in good $\gamma$-sensitivity but the worst figure of merit. Reducing the measurement duration to match the fastest algorithm, time to $10 \%$, resulted in the tail sum method having the best figure of merit and the best $\gamma$-sensitivity of approximately $6 \times 10^{-7}$ counts per gamma. It should be noted that the accuracy of the $\gamma$-sensitivity results for the best tail sum measurement is limited by the counting statistics of the number of $y$-rays.

It is also worth noting that for similar figure of merit, the tail sum algorithm had a lower $\gamma$-sensitivity than the charge integration method. Given that the figure of merit is a measure of the overlap in the statistical distribution of both types of radiation, the same figure of merit should result in the same $\mathrm{Y}$-sensitivity. A possible explanation in this discrepancy is that the assumption that data can be described by a Gaussian is incorrect. Prusachenko et al. state that the assumption that the distributions are Gaussian can only be made where there are no singularities from one region to another [13]. Noise in the system, events such as direct hits to the PMT and pileup also appear as events in the PSD and FoM plots which would create singularities from one region to another.

Table 1: Summary of performance for each algorithm with settings optimized for the best figure of merit and settings corresponding to duration of the time to $10 \%$ algorithm.

\begin{tabular}{c||ccccc}
\hline \hline \multirow{2}{*}{ Algorithm } & Optimization & Figure of & V-sensitivity & Theoretical \\
& Merit & $\left(10^{-6}\right.$ counts & Duration* & Count Rate \\
(counts per & pergamma) & second) \\
\hline \hline
\end{tabular}




\begin{tabular}{|c|c|c|c|c|c|}
\hline Charge integration & $\begin{array}{c}\text { Figure of } \\
\text { Merit }\end{array}$ & $1.37 \pm 0.008$ & $2.0 \pm 0.66$ & 272.5 & $3.67 \times 10^{6}$ \\
\hline $\begin{array}{c}\text { Filtered Charge } \\
\text { integration }\end{array}$ & $\begin{array}{c}\text { Figure of } \\
\text { Merit }\end{array}$ & $1.32 \pm 0.012$ & $5.0 \pm 1$ & 267.5 & $3.74 \times 10^{6}$ \\
\hline Tail sum & $\begin{array}{c}\text { Figure of } \\
\text { Merit }\end{array}$ & $1.36 \pm 0.014$ & $\leq 0.1 \pm 0.59$ & 250 & $4.00 \times 10^{6}$ \\
\hline Charge integration & Count Rate & $1.21 \pm 0.004$ & $7.0 \pm 1.1$ & 125 & $8 \times 10^{6}$ \\
\hline $\begin{array}{c}\text { Filtered Charge } \\
\text { integration }\end{array}$ & Count Rate & $1.21 \pm 0.004$ & $7.0 \pm 1.0$ & 125 & $8 \times 10^{6}$ \\
\hline Tail sum & Count Rate & $1.26 \pm 0.009$ & $0.6 \pm 0.69$ & 125 & $8 \times 10^{6}$ \\
\hline Time to $10 \%$ & N/A & $1.11 \pm 0.035$ & $3.0 \pm 0.86$ & $125 \pm 14$ & $8 \times 10^{6}$ \\
\hline
\end{tabular}

* Errors on the duration for all algorithms apart from time to $10 \%$ is $\pm 2.5 \mathrm{~ns}$ which is the length of one sample.

A summary of the performance of each algorithm is displayed in table 1. Results are displayed for the optimization for best figure of merit and for duration that matched the time to $10 \%$ in count rate. The best gamma sensitivity of approximately $10^{-7}$ is achieved with the tail sum method optimized for rejecting gammas. The high rate optimization was taken to be the same measurement duration corresponding to the average time to $10 \%$ for the neutron pulses. Table 1 includes a simplified measure indicative of the upper limit of count rate capability. The theoretical count rate shown can only be achieved given a regular, repetitive pulse whereas the signals of interest are much more random. The actual count rate achievable with each algorithm can be up to a factor of 10 smaller and will be investigated in the future.

\subsection{Performance at high incident neutron rates}

The 2D pulse shape histograms of data taken at a peak neutron rate of $960 \mathrm{knps}$ using the charge integration method and the tail sum methods are shown in figure $7 \mathrm{c}$ and $7 \mathrm{~d}$ respectively. The tail sum method was used over the time to $10 \%$ due to its compromise between superior gamma sensitivity and short duration as discussed in section 4.1. Here it is obvious that the thermal neutron peak is better separated from the gamma when using the tail sum method. No fast neutrons are visible because they are rejected by time of flight on the EMMA beamline.

Several interesting artefacts related to pulse pile-up can be seen in figure 7 that are not present in the PSD plots for the data taken on the ${ }^{241} \mathrm{Am}$-Be source (figure 3). There are four possibilities of pileup on the EMMA beamline: neutron-neutron, gamma-gamma, neutron-gamma and gammaneutron. The neutron-neutron pileup events are most probable at high incident neutron rates while the gamma-gamma events are probable at low incident neutron rates due to $(n, \gamma)$ reactions in the shielding.

Pileup artefacts manifest in different ways for the two PSD algorithms depending on the arrival time of the second event with respect to the first. In the charge integration method, if the second pulse arrives within the short window, then the PSD is reduced leading to the regions observed below the standard gamma continuum in figure 7 a due to pulse height being determined by the type of 
radiation arriving first. If, for example, a neutron arrives followed by a gamma within the short window, then the pulse height is measured to be that of the neutron but the PSD value is significantly reduced because of the large $Q_{s}$ leading to the small island region highlighted by the black dashed line box located around $0.07 \mathrm{~V}$ and 0.3 a.u. for the PSD value. If the arrival time of the second event is outside the short window but within the long window, the PSD value of the charge integration will increase thus leading to the extra events observed above the gamma continuum and outside the thermal neutron peak. Again, the type of the initial radiation determines the pulse height. Therefore, the line of events extending vertically from the thermal neutron peak in figure $7 \mathrm{a}$, highlighted by the black dotted square all contain at least one neutron. Likewise, any event outside of the thermal neutron pulse height window contains at least one gamma event. The type of the second interaction is, however, entirely ambiguous using these methods. The probability of a second event occurring outside the short window and within the long window is significantly higher than two events occurring within the short window accounting for the larger number of events above the gamma continuum.
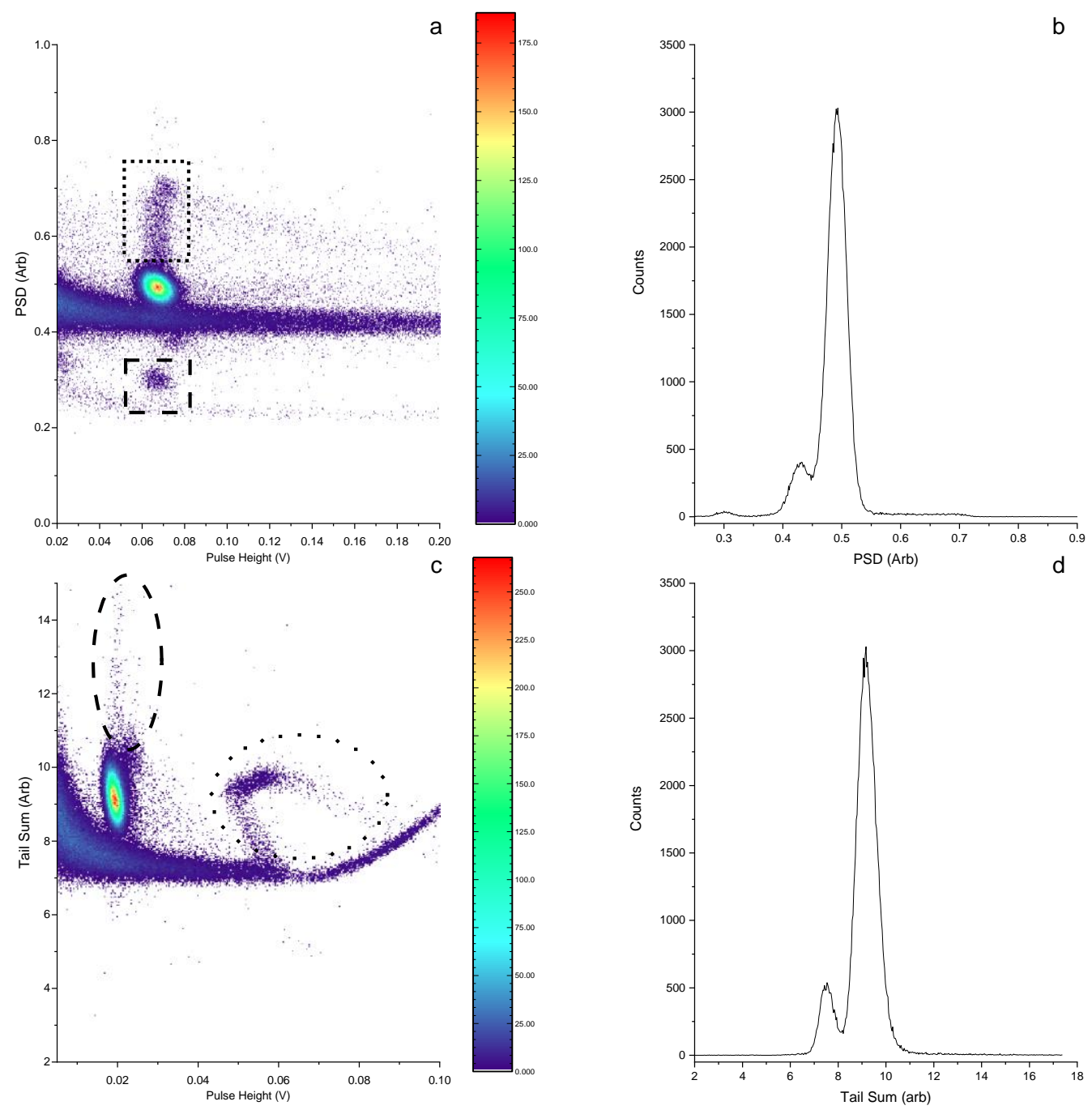
Figure 7: PSD Plots for the for the (a) charge integration and (c) tail sum algorithms optimized for count rate along with corresponding cuts across the PSD axis for the energy corresponding to thermal neutrons shown in (b) for charge integration and (c) for tail sum at peak incident rate 960 knps at the EMMA beamline at ISIS.

Pileup artefacts for the tail sum method will only result in larger PSD values owing to integrating in only a single short window. This short duration of integration also means that the number of pileup events is significantly reduced over the charge integration method. Because the re-trigger time of the tail sum method is shorter, events that would have occurred within the long window are counted as separate events. These will, however, suffer from baseline issues. The pulse height of these events will be larger compared to those that are well separated in time because the event occurs on the tail of the scintillator emission. The thermal neutron peak is therefore broadened in pulse height as seen in figure $7 \mathrm{c}$. If the second event occurs within the tail sum integration time, then the PSD value will increase (eq 4) giving rise to the vertical line of events extending from the thermal neutron peak, highlighted by the black dotted ellipse, and also the slight reduction of FoM illustrated in figure $7 \mathrm{~d}$. Events with pulse height greater than $0.04 \mathrm{~V}$ in figure $7 \mathrm{c}$ are a result of the pulses clipping on the ADC and subsequent smearing of the signal by the Bessel filter. In this case, the tail sum increases rapidly while there is little change in the filtered pulse height. Clipped pulses with pileup are manifested in the region with pulse height greater than $0.04 \mathrm{~V}$ and tail sum value above the standard gamma continuum, as highlighted by the black dotted circle. The slope and position of those features are dependent on the sample at which the tail sum starts and the number of samples integrated over. Further investigations on the tail sum algorithm performance at high rate will be carried out and reported on in subsequent papers.

\section{Conclusion}

The new ${ }^{6} \mathrm{Li}$ loaded plastic scintillator EJ-270 has clear potential for high count rate applications when coupled with a negative polarity PMT base. The performance of EJ-270 was investigated along with four different pulse shape discrimination algorithms. The tail sum method was found to give the best results when the measurement duration of the algorithms were set to match the time to $10 \%$. The figure of merit for this duration of $125 \mathrm{~ns}$ was 1.26 and the $\gamma$-sensitivity was $6 \times 10^{-7}$. The addition of a digital low pass filter to the charge integration method was found to reduce the best achievable figure of merit and $\gamma$-sensitivity but resulted in a slight increase in figure of merit for the short duration measurements.

The tail sum algorithm developed here to exploit the high count rate potential of EJ-270 is mathematically simple, and can be easily implemented into an FPGA for real time high rate applications. The tail sum algorithm outperformed the traditional charge integration algorithm in terms of $\gamma$ sensitivity, and ultimate rate capability. The charge integration method had the best figure of merit when a long duration sample window was used. However, when pushing the limits of the algorithms in terms of short measurement windows the tail sum method was found to give the best figure of merit and $\gamma$-sensitivity. The implementation of the tail sum algorithm an FPGA will be the subject of a subsequent publication.

\section{Acknowledgements}


This work was funded by the Science and Technology Facilities Council (STFC) through the ISIS department and the Centre for Instrumentation (CFI). We would also like to thank Matt Wilson and Paul Seller of the STFC's Technology Department's Detector Development Group, Nigel Rhodes and Erik Schooneveld of STFC's ISIS Neutron Detector Group and Paul Sellin at the University of Surrey for supporting this work. We would also like to thank the author of the PeakUtils python library.

\section{References}

[1] I. Stefanescu, M. Christensen, J. Fenske, R. Hall-Wilton, P.F. Henry, O. Kirstein, M. Müller, G. Nowak, D. Pooley, D. Raspino, N. Rhodes, J. Šaroun, J. Schefer, E. Schooneveld, J. Sykora, W. Schweika, Neutron detectors for the ESS diffractometers, J. Inst. 12 (2017) P01019-P01019. https://doi.org/10.1088/1748-0221/12/01/P01019.

[2] N.J. Rhodes, E.M. Schooneveld, R.S. Eccleston, Current status and future directions of position sensitive neutron detectors at ISIS, Nuclear Instruments and Methods in Physics Research Section A: Accelerators, Spectrometers, Detectors and Associated Equipment. 529 (2004) 243-248. https://doi.org/10.1016/j.nima.2004.04.152.

[3] N. Zaitseva, A. Glenn, H. Paul Martinez, L. Carman, I. Pawełczak, M. Faust, S. Payne, Pulse shape discrimination with lithium-containing organic scintillators, Nuclear Instruments and Methods in Physics Research Section A: Accelerators, Spectrometers, Detectors and Associated Equipment. 729 (2013) 747-754. https://doi.org/10.1016/j.nima.2013.08.048.

[4] N.J. Cherepy, R.D. Sanner, P.R. Beck, E.L. Swanberg, T.M. Tillotson, S.A. Payne, C.R. Hurlbut, Bismuth- and lithium-loaded plastic scintillators for gamma and neutron detection, Nucl. Instruments Methods Phys. Res. Sect. A Accel. Spectrometers, Detect. Assoc. Equip. 778 (2015) 126132. https://doi.org/10.1016/j.nima.2015.01.008.

[5] M. Loyd, M. Pianassola, C. Hurlbut, K. Shipp, L. Sideropoulos, K. Weston, M. Koschan, C.L. Melcher, M. Zhuravleva, Effects of temporary fogging and defogging in plastic scintillators, Nucl. Instruments Methods Phys. Res. Sect. A Accel. Spectrometers, Detect. Assoc. Equip. 922 (2019) 202208. https://doi.org/10.1016/j.nima.2018.12.087.

[6] S. Richards, G.J. Sykora, X-ray induced radiation damage in CLYC(Ce), Nucl. Instruments Methods Phys. Res. Sect. A Accel. Spectrometers, Detect. Assoc. Equip. 902 (2018) 45-50. https://doi.org/10.1016/i.nima.2018.06.045.

[7] J. Glodo, R. Hawrami, K.S. Shah, Development of Cs2LiYCl6 scintillator, J. Cryst. Growth. 379 (2013) 73-78. https://doi.org/10.1016/i.jcrysgro.2013.03.023.

[8] N. Zaitseva, B.L. Rupert, I. PaweŁczak, A. Glenn, H.P. Martinez, L. Carman, M. Faust, N. Cherepy, S. Payne, Plastic scintillators with efficient neutron/gamma pulse shape discrimination, Nucl. Instruments Methods Phys. Res. Sect. A Accel. Spectrometers, Detect. Assoc. Equip. 668 (2012) 8893. https://doi.org/10.1016/i.nima.2011.11.071. 
[9] M. Flaska, S.A. Pozzi, Identification of shielded neutron sources with the liquid scintillator BC501A using a digital pulse shape discrimination method, Nucl. Instruments Methods Phys. Res. Sect. A Accel. Spectrometers, Detect. Assoc. Equip. 577 (2007) 654-663.

https://doi.org/10.1016/j.nima.2007.04.141.

[10] S.D. Clarke, M. Flaska, S.A. Pozzi, P. Peerani, Neutron and gamma-ray cross-correlation measurements of plutonium oxide powder, Nucl. Instruments Methods Phys. Res. Sect. A Accel. Spectrometers, Detect. Assoc. Equip. 604 (2009) 618-623. https://doi.org/10.1016/i.nima.2009.02.045.

[11] S.A. Pozzi, S.D. Clarke, M. Flaska, P. Peerani, Pulse-height distributions of neutron and gamma rays from plutonium-oxide samples, Nucl. Instruments Methods Phys. Res. Sect. A Accel. Spectrometers, Detect. Assoc. Equip. 608 (2009) 310-315. https://doi.org/10.1016/i.nima.2009.07.007.

[12] G.F. Knoll, Radiation Detection and Measurement, 4th ed., John Wiley and Sons, 2010.

[13] P.S. Prusachenko, V.A. Khryachkov, V. V. Ketlerov, M. V. Bokhovko, I.P. Bondarenko, Optimization of the $n / \gamma$ separation algorithm for a digital neutron spectrometer, Nucl. Instruments Methods Phys. Res. Sect. A Accel. Spectrometers, Detect. Assoc. Equip. 905 (2018) 160-170. https://doi.org/10.1016/j.nima.2018.07.062.

[14] G. J. Sykora, EMMA operating modes, STFC internal report (2015).

[15] S. Van Der Walt, S.C. Colbert, G. Varoquaux, The NumPy array: A structure for efficient numerical computation, Comput. Sci. Eng. 13 (2011) 22-30. https://doi.org/10.1109/MCSE.2011.37.

[16] P. Virtanen, R. Gommers, T.E. Oliphant, M. Haberland, T. Reddy, D. Cournapeau, E. Burovski, P. Peterson, W. Weckesser, J. Bright, S.J. van der Walt, M. Brett, J. Wilson, K.J. Millman, N. Mayorov, A.R.J. Nelson, E. Jones, R. Kern, E. Larson, C.J. Carey, I. Polat, Y. Feng, E.W. Moore, J. VanderPlas, D. Laxalde, J. Perktold, R. Cimrman, I. Henriksen, E.A. Quintero, C.R. Harris, A.M. Archibald, A.H. Ribeiro, F. Pedregosa, P. van Mulbregt, A. Vijaykumar, A. Pietro Bardelli, A. Rothberg, A. Hilboll, A. Kloeckner, A. Scopatz, A. Lee, A. Rokem, C.N. Woods, C. Fulton, C. Masson, C. Häggström, C. Fitzgerald, D.A. Nicholson, D.R. Hagen, D. V. Pasechnik, E. Olivetti, E. Martin, E. Wieser, F. Silva, F. Lenders, F. Wilhelm, G. Young, G.A. Price, G.L. Ingold, G.E. Allen, G.R. Lee, H. Audren, I. Probst, J.P. Dietrich, J. Silterra, J.T. Webber, J. Slavič, J. Nothman, J. Buchner, J. Kulick, J.L. Schönberger, J.V. de Miranda Cardoso, J. Reimer, J. Harrington, J.L.C. Rodríguez, J. Nunez-Iglesias, J. Kuczynski, K. Tritz, M. Thoma, M. Newville, M. Kümmerer, M. Bolingbroke, M. Tartre, M. Pak, N.J. Smith, N. Nowaczyk, N. Shebanov, O. Pavlyk, P.A. Brodtkorb, P. Lee, R.T. McGibbon, R. Feldbauer, S. Lewis, S. Tygier, S. Sievert, S. Vigna, S. Peterson, S. More, T. Pudlik, T. Oshima, T.J. Pingel, T.P. Robitaille, T. Spura, T.R. Jones, T. Cera, T. Leslie, T. Zito, T. Krauss, U. Upadhyay, Y.O. Halchenko, Y. Vázquez-Baeza, SciPy 1.0: fundamental algorithms for scientific computing in Python, Nat. Methods. 17 (2020) 261-272. https://doi.org/10.1038/s41592-019-0686-2. 
421 [17] R.A. Winyard, J.E. Lutkin, G.W. McBeth, Pulse shape discrimination in inorganic and organic 422 scintillators. I, Nucl. Instruments Methods. 95 (1971) 141-153. https://doi.org/10.1016/0029$423554 \times(71) 90054-1$. [18] R. Kouzes, J.R. Ely, A.T. Lintereur, D.L. Stephens. Neutron Detector Gamma Insensitivity Criteria. Pacific Northwest National Laboratory report PNNL-18903. (2009) 